\title{
Survey on Cloud Based Robotics Architecture, Challenges and Applications
}

\author{
Dr. Subarna Shakya \\ Professor, \\ Department of Electronics and Computer Engineering \\ Central Campus, Institute of Engineering, Pulchowk \\ Tribhuvan University, Pulchowk \\ Lalitpur Nepal. \\ Email: drss@ioe.edu.np
}

\begin{abstract}
The emergence of the cloud computing, and the other advanced technologies has made possible the extension of the computing and the data distribution competencies of the robotics that are networked by developing an cloud based robotic architecture by utilizing both the centralized and decentralized cloud that is manages the machine to cloud and the machine to machine communication respectively. The incorporation of the robotic system with the cloud makes probable the designing of the cost effective robotic architecture that enjoys the enhanced efficiency and a heightened real- time performance. This cloud based robotics designed by amalgamation of robotics and the cloud technologies empowers the web enabled robots to access the services of cloud on the fly. The paper is a survey about the cloud based robotic architecture, explaining the forces that necessitate the robotics merged with the cloud, its application and the major concerns and the challenges endured in the robotics that is integrated with the cloud. The paper scopes to provide a detailed study on the changes influenced by the cloud computing over the industrial robots.
\end{abstract}

Keywords: Robotics, Cloud Computing, Cloud Based Robotics, Big Data, Internet of Things, Open Source, Challenges and Applications

\section{Introduction}

The robotics that plays a vital role in automating the daily routines that are carried out regularly, the progress in the robots development has increased at a rapid pace in the industries and this has caused the robots that are programmed to reach a heightened performance in the real time providing a better compatibility, accuracy and robustness. The network framed using robots is the cluster of robots, where every robots I it is referred to as a node or a data point, these cluster of robots are linked using the either the wired or wireless communication. The robots are comprised of number sensing elements and the capabilities of decision making to perform the tasks in a more proficient way. The developments of the more advanced technologies have made the robotics to be utilized in a wide range of application such as the "analytical services, global

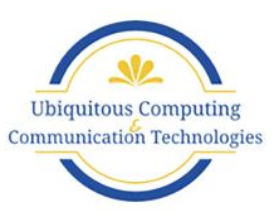


Journal of Ubiquitous Computing and Communication Technologies (UCCT) (2020)

Vol.02/ No. 01

Pages: $10-18$

https://www.irojournals.com/jucct/

DOI: https://doi.org/10.36548/jucct.2020.1.002

positioning system, artificial intelligence biometric sensors, language processing, and atmospheric sensing and so on. But the memory in the robots are insufficient and very low for processing and storing the information's, to bring down the severity in the processing and storing the Robotics are integrated with the cloud that renders an on demand service to the devices such as robots [1-2].

The cloud delivers services on pay as you go terms to the devices as well as the collective group of people. These could be categorized based type of services provided by the cloud as the software as service also known as the SaaS, infrastructure as services-IaaS and the platform as services-PaaS all these services rendered by the cloud are hosted by the cloud from the servers of the provider and the propelled into the users device based on their request. Enormous storage capacities and the elastic nature of the cloud computing makes it compatible for a wide range of industrial and business applications. The cloud finds it way in the robotics as the idea behind the traditional standalone robots and the networked robots were well suited, cost effective and efficient for the static environment and were a failure when used in the dynamic environment with the scalable requirements due to their limitations endured in the manipulations done on board. However the rapid advancements and the newly emerging technologies have made the robots up gradation essential. This paved way for the merging of the cloud computing with the robotics to form a novel term cloud robotics as the sharing of resources and the cost effectiveness was the important issues that are to be taken into consideration. The concept behind the development was basically to bring down the production cost and enhance the throughput. The figure.1 below shows the three strategies of cloud robotics [3-5].

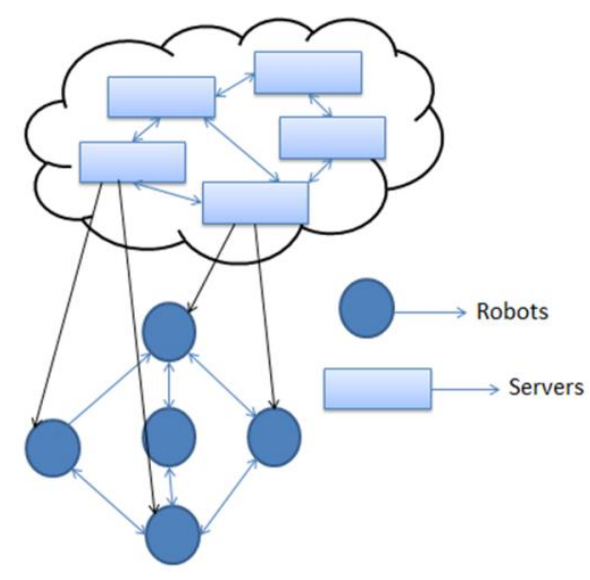

Figure 1. (a) Peer based Frame Work for Computation 
Journal of Ubiquitous Computing and Communication Technologies (UCCT) (2020)

Vol.02/ No. 01

Pages: $10-18$

https://www.irojournals.com/jucct/

DOI: https://doi.org/10.36548/jucct.2020.1.002

In Figure 1. (a) Peer based Frame Work for Computation the robotic network are directly connected to the cloud paradigm and provides a moderate robustness and interoperability with the high mobility

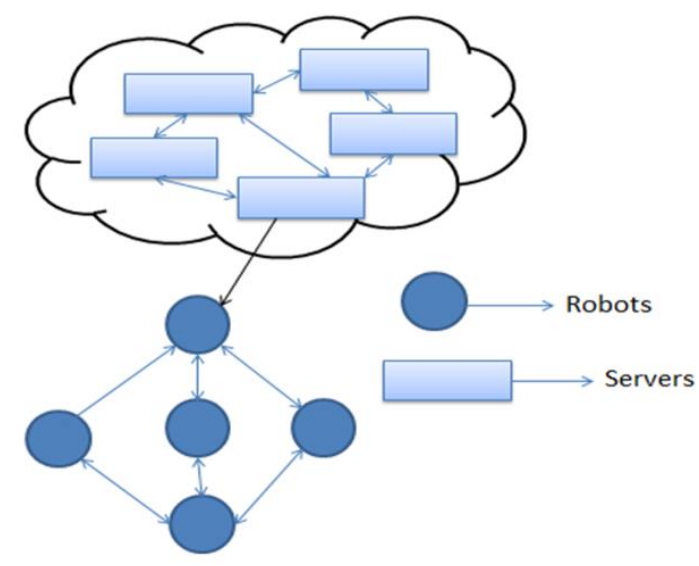

Figure 1. (b) Proxy based Frame Work for Computation

In Figure 1. (b) Proxy based frame work "the VM unit and the Robotic network in the cloud paradigm masters and maintains the bridge in receiving and distributing the task in the collaborative manner" providing a lower level of robustness with high interoperability and medium mobility.

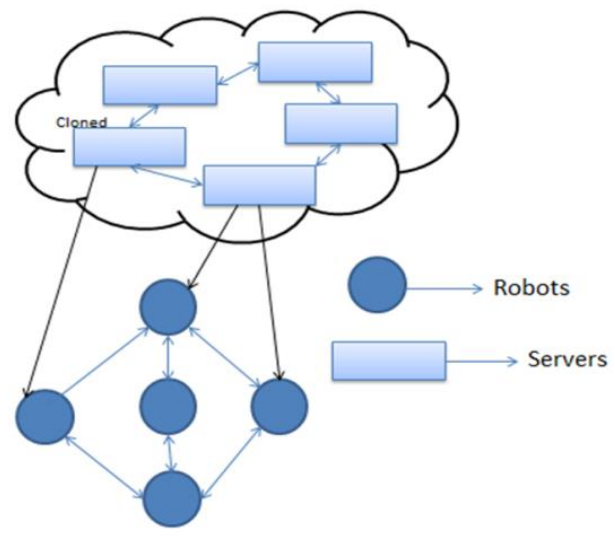

Figure 1. (c)Clone based Frame Work for Computation 
Whereas in Figure 1. (c) Clone based frame work for computation "where each and every robot has a copy or clone in the cloud and the jobs are assigned to the robot itself or to its clone". The clone based provides a heightened robustness with the low interoperability and low mobility. Every computational architecture has its own merits and demerits in the varying scenarios, "The models are always implemented depending on the system resource availability, application requirements and state of networks on the basis of the amount of the work load"[7-9].

The paper organizes a survey on the cloud based robotic architecture, studying the need and the driving force behind the robotic architecture and further presenting the challenges endured and the applications of the robotics, with the complete study of the cloud robotics architecture in section 2, The Enabling forces of Cloud Robotics 3, the major concerns and the challenges endured in cloud robotics in section 4, the cloud robotics applications in section 5 and conclusion in section 6 .

\section{Cloud Robotics Architecture}

The robotics that is networked is visualized as a state of transformation from the robots that are programmed to robot enabled with the clouds. The aim in developing the cloud robotics is to offload its highly complex computations to the cloud paradigm and minimizes the load forced over the each robot, This architecture mainly consists of two sections, or even can be split as two layers bottom layer and top layer, where the bottom layer holds different types of robots and the top layer is the cloud platform with its supporting components such as the proxy servers, massive spatial database, high-performance servers and other related components. The figure. 2 below shows the general architecture of the cloud based robotics in an industrial environment [10].

Where the robot to robot communication is framed simply in the adhoc manner and the robot to cloud is framed in a centralized manner. The communications extended between the robots are carried out in a wireless medium forming a collaborative computing. This is initiated by developing pooled robots forming an adhoc cloud paradigm followed by the information transfer to the robots pooled. This causes even the robots that beyond the cloud access point to utilize the information's from the cloud. Further the infrastructures of the cloud are enables all the pooled robots to enjoy the resources of the cloud based on their demand.

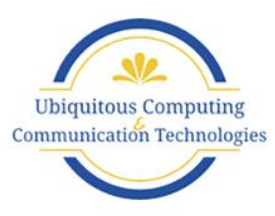




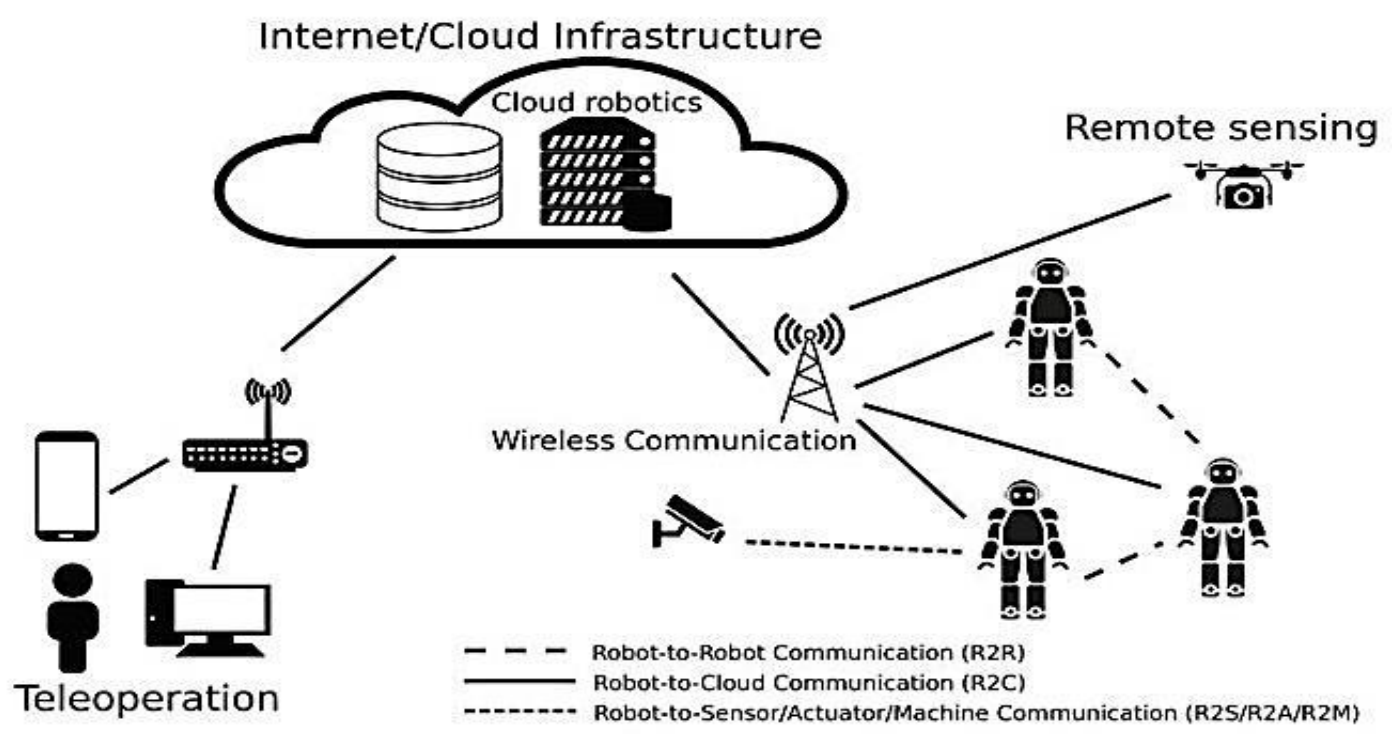

Figure.2 General Cloud Robotics in an Industrial Environment [10]

Thus allowing the clustered robots to offload computational- intensive tasks into the cloud platform for the execution to be carried out in a remote location developing robots with the "remote brain" the main advantages of the cloud based robotics architecture are, the robots are facilitated with the large volume of storage and equipped with the broad library skills that are required to perform the task.

\section{The Enabling Forces of Cloud Robotics}

The cloud robotics are enabled with the multiple of resources that are provided by the cloud networking and other technologies along with the communication protocols that are wireless. The section provides the detailed picture of the various enabling forces of cloud based robotics. The primary enabling force of cloud robotics is the cloud computing that has enhanced the rapidity of robots and the automated equipment's development. In paper [11] the SLAM utilizing the plat from of cloud was proposed to have qualitative results. Further the future cloud based robotics would concentrate more in analyzing the video, image, data mining and many other fields. The next enabling force is the big data providing a system that is automated by accessing huge resources from the infrastructure of the cloud limiting the challenges of the resources on board [12] the insights gained from the big data offers significant technical support that are essential in the development of the navigation achieved through the robots and as well as the system used in grasping. The next essential driving force is the open source, cooperative learning that is empowered by the learning

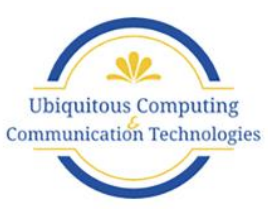


Journal of Ubiquitous Computing and Communication Technologies (UCCT) (2020)

Vol.02/ No. 01

Pages: $10-18$

https://www.irojournals.com/jucct/

DOI: https://doi.org/10.36548/jucct.2020.1.002

techniques. They are the Reinforcement learning [13], Q-learning [14], hierarchical reinforcement learning, the learning techniques in the robotic network enhances the co-operative learning in the networked robotics enabling them to tackle challenging problems and importantly enhancing the accuracy in performing the tasks. The final enabling force is the network connectivity that is developed using the adhoc and the proactive routing methodologies [15]. The figure.3 below provides the routing protocol followed in the mobile robotic network [16].

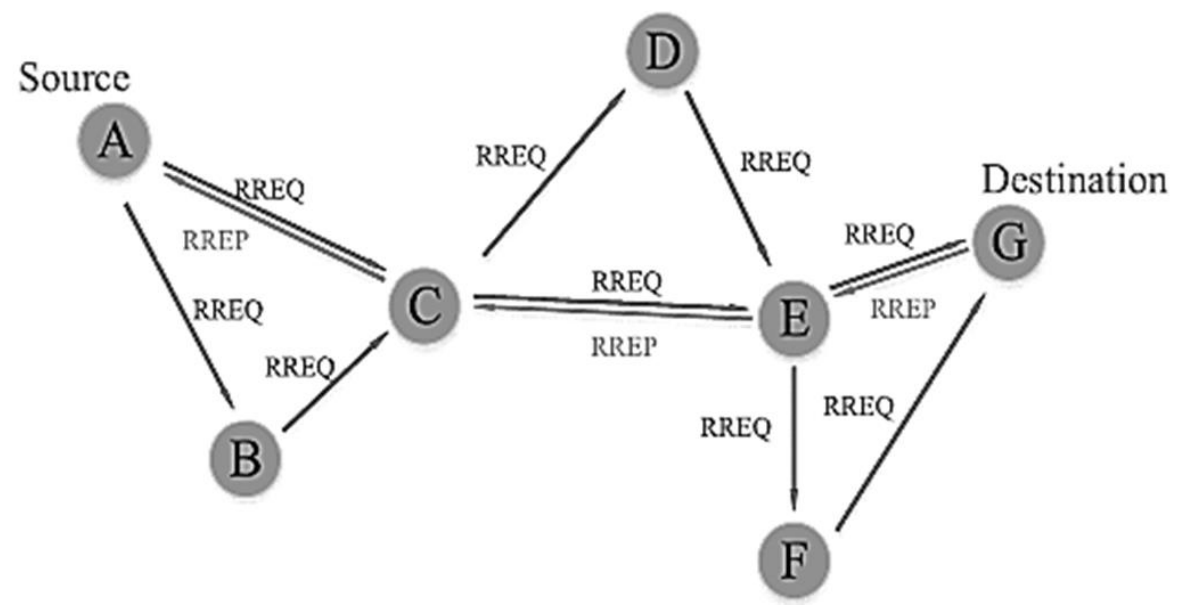

Figure.3 Classical Routing Protocol [16]

\section{The Major Concerns and Challenges Endured In Cloud Robotics}

This section provides the major concerns and the challenges endured in the cloud computing. The table. 1 below provides the various challenges suffered by the cloud computing and its descriptions

\begin{tabular}{|c|c|c|}
\hline Challenges & Description & Solutions \\
\hline $\begin{array}{l}\text { Resource } \\
\text { Allocation And } \\
\text { Scheduling [16] } \\
{[17]}\end{array}$ & $\begin{array}{l}\text { "As offloading the computational tasks to the } \\
\text { cloud is a notable characteristics of the cloud" } \\
\text { "Taking into consideration the different working } \\
\text { equipment, interface settings, and network } \\
\text { environments, for a given computational task, the } \\
\text { choice of uploading, self-processing or assigning }\end{array}$ & $\begin{array}{l}\text { "Developing protocols } \\
\text { enabling careful selection of } \\
\text { resources, offloading schemes, } \\
\text { with the capability of } \\
\text { managing delay incurred". }\end{array}$ \\
\hline
\end{tabular}

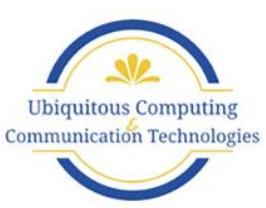




\begin{tabular}{|c|c|c|}
\hline & $\begin{array}{l}\text { the task to the nearest node has an important } \\
\text { impact on overall performance". } \\
\text { "Some of the challenges endured are delay, } \\
\text { compatible resource allocation and carefully } \\
\text { selection of offloading schemes." }\end{array}$ & \\
\hline $\begin{array}{l}\text { Data } \\
\text { Interaction } \\
\text { Between Robot } \\
\text { And Cloud } \\
\text { Platform [18] }\end{array}$ & $\begin{array}{l}\text { "Faces challenges such as requirements of } \\
\text { multiple interfaces equipped with various data } \\
\text { formats, requires proper preprocessing, } \\
\text { robustness , compatibility problems", }\end{array}$ & $\begin{array}{l}\text { "The data interaction between } \\
\text { the cloud and robotics could } \\
\text { be improved employing the } \\
\text { Software defined Networks". }\end{array}$ \\
\hline $\begin{array}{l}\text { Security In } \\
\text { Cloud [19] [20] } \\
{[21]}\end{array}$ & $\begin{array}{l}\text { "These are hidden dangers affecting the devices } \\
\text { used in computing and sensing, causing serious } \\
\text { data leakages and loss of key data" }\end{array}$ & $\begin{array}{l}\text { "Utilize authenticating } \\
\text { schemes by incorporating } \\
\text { the cryptographic techniques } \\
\text { that are symmetric and } \\
\text { Asymmetric" }\end{array}$ \\
\hline
\end{tabular}

Table.1 Summarizes the Challenges Endured and it's Solution

\section{Cloud Robotics Applications}

The cloud based robotics was mainly used in applications like SLAM "that are utilized in breaking the bottleneck caused by the limited onboard computing and the storage equipment" grasping system that are used in identifying the unknown objects and navigation presently the telerobotics [22] based on the cloud computing are employed in multiple of applications such as home automation, medical care, military purpose etc. The artificial integration in the cloud based robotics [23] would further enhance the performance of the system by improving the automation process, in terms of delay [24], processing speed [25] and access control.

\section{Conclusion}

The paper is a complete study on the cloud robotics, presenting the overview on the particulars of the architecture of the cloud robotics, the enabling forces of the cloud robotics, concerns and the challenges related to the cloud robotics and some of the applications associated with the cloud robotics. The paper highlights the different architectures of the robotic cloud computing, and presents the solution for the challenges endured by it. In future the paper aims in developing a cloud based robotics for the improving the primary health care services in the underdeveloped areas that mostly affected by epidemic disease.

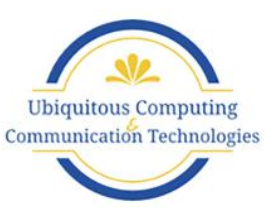


Journal of Ubiquitous Computing and Communication Technologies (UCCT) (2020)

Vol.02/ No. 01

Pages: $10-18$

https://www.irojournals.com/jucct/

DOI: https://doi.org/10.36548/jucct.2020.1.002

\section{References}

[1] Chowdhury, Akash, Swastik Mukherjee, and Sourav Banerjee. "An Approach towards Survey and Analysis of Cloud Robotics." Detecting and Mitigating Robotic Cyber Security Risks (2017): 208.

[2] Abou Allaban, Anas, Maozhen Wang, and Taşkın Padır. "A Systematic Review of Robotics Research in Support of In-Home Care for Older Adults." Information 11, no. 2 (2020): 75.

[3] Pakkala, Daniel, Jani Koivusaari, Pekka Pääkkönen, and James Spohrer. "An Experimental Case Study on Edge Computing based Cyber-Physical Digital Service Provisioning with Mobile Robotics." In Proceedings of the 53rd Hawaii International Conference on System Sciences. 2020.

[4] Toffetti, Giovanni, and Thomas Michael Bohnert. "Cloud Robotics with ROS." In Robot Operating System (ROS), pp. 119-146. Springer, Cham, 2020.

[5] Urrea, Claudio, and Rodrigo Matteoda. "Development of a virtual reality simulator for a strategy for coordinating cooperative manipulator robots using cloud computing." Robotics and Autonomous Systems 126 (2020): 103447.

[6] Ichnowski, Jeffrey, Jan Prins, and Ron Alterovitz. "The Economic Case for Cloud-Based Computation for Robot Motion Planning." In Robotics Research, pp. 59-65. Springer, Cham, 2020.

[7] da Silva Pereira, Diego, Bruno Agenor Santana, Rosiery Silva Maia, and Anderson Souza. "A cloud robotics architecture clone based for a cellbots team." IEEE Latin America Transactions 15, no. 9 (2017): 1587-1594.

[8] Aagela, Hamza. "CCRP: A Novel Clone-Based Cloud Robotic Platform for Multi-Robots." PhD diss., University of Huddersfield, 2019.

[9] Li, Gaofeng, Hongpeng Wang, Xin Ying, and Jingtai Liu. "A proxy-based cloud infrastructure for home service robots." In The 27th Chinese Control and Decision Conference (2015 CCDC), pp. 5718-5723. IEEE, 2015.

[10] Barriquello, Carlos Henrique, F. E. S. Silva, Daniel Pinheiro Bernardon, Luciane Neves Canha, M. J. D. S. Ramos, and Daniel Sperb Porto. "Fundamentals of Wireless Communication Link Design for Networked Robotics." Service Robots (2018): 127-142.

[11] Riazuelo, Luis, Moritz Tenorth, Daniel Di Marco, Marta Salas, Dorian Gálvez-López, Lorenz Mösenlechner, Lars Kunze et al. "RoboEarth semantic mapping: A cloud enabled knowledgebased approach." IEEE Transactions on Automation Science and Engineering 12, no. 2 (2015): 432-443.

[12] Yuan, Wei, Pan Deng, Tarik Taleb, Jiafu Wan, and Chaofan Bi. "An unlicensed taxi identification model based on big data analysis." IEEE Transactions on Intelligent Transportation Systems 17, no. 6 (2015): 1703-1713.

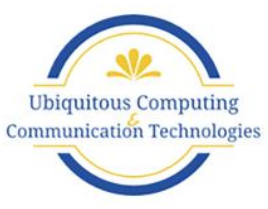


Journal of Ubiquitous Computing and Communication Technologies (UCCT) (2020)

Vol.02/ No. 01

Pages: $10-18$

https://www.irojournals.com/jucct/

DOI: https://doi.org/10.36548/jucct.2020.1.002

[13] Wang, Yang, and Clarence W. De Silva. "An object transportation system with multiple robots and machine learning." In Proceedings of the 2005, American Control Conference, 2005., pp. 1371-1376. IEEE, 2005.

[14] Wang, Ying, and Clarence W. de Silva. "Extend single-agent reinforcement learning approach to a multi-robot cooperative task in an unknown dynamic environment." In The 2006 IEEE International Joint Conference on Neural Network Proceedings, pp. 4999-5005. IEEE, 2006.

[15] Agüero, Carlos E., Nate Koenig, Ian Chen, Hugo Boyer, Steven Peters, John Hsu, Brian Gerkey et al. "Inside the virtual robotics challenge: Simulating real-time robotic disaster response." IEEE Transactions on Automation Science and Engineering 12, no. 2 (2015): 494506.

[16] Gouveia, Bruno Duarte, David Portugal, Daniel C. Silva, and Lino Marques. "Computation sharing in distributed robotic systems: A case study on SLAM." IEEE Transactions on Automation Science and Engineering 12, no. 2 (2014): 410-422.

[17] Salmerón-Garc1, Javier, Pablo Inigo-Blasco, Fernando D1, and Daniel Cagigas-Muniz. "A tradeoff analysis of a cloud-based robot navigation assistant using stereo image processing." IEEE Transactions on Automation Science and Engineering 12, no. 2 (2015): 444454.

[18] Shu, Zhaogang, Jiafu Wan, Di Li, Jiaxiang Lin, Athanasios V. Vasilakos, and Muhammad Imran. "Security in software-defined networking: Threats and countermeasures." Mobile Networks and Applications 21, no. 5 (2016): 764-776.

[19] Chen, Min, Yin Zhang, Yong Li, Shiwen Mao, and Victor CM Leung. "EMC: Emotion-aware mobile cloud computing in 5G." IEEE Network 29, no. 2 (2015): 32-38.

[20] Chen, Min. "NDNC-BAN: supporting rich media healthcare services via named data networking in cloud-assisted wireless body area networks." Information Sciences 284 (2014): 142-156.

[21] Chen, Min, Yixue Hao, Yong Li, Chin-Feng Lai, and Di Wu. "On the computation offloading at ad hoc cloudlet: architecture and service modes." IEEE Communications Magazine 53, no. 6 (2015): 18-24.

[22] Manoharan, S., \& Ponraj, N. (2019). Precision improvement and delay reduction in surgical telerobotics. Journal of Artificial Intelligence, 1(01), 28-36.

[23] Smys, S., \& Ranganathan, G. (2019). Robot assisted sensing, control and manufacture in automobile industry. Journal of ISMAC, 1(03), 180-187.

[24] Valanarasu, M. R. (2019). Smart and secure iot and ai integration framework for hospital environment. Journal of ISMAC, 1(03), 172-179.

[25] Vijayakumar, T. (2019). Flexible robotic electronic skin with high sensitivity sensor arrays. Journal of Electronics, 1(01), 43-51.

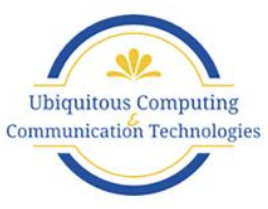

